\title{
The influence of primary care physicians' mental health knowledge, attitudes and self-efficacy on referrals to specialised services: findings from a longitudinal pilot trial
}

Jessica Spagnolo, Helen-Maria Vasiliadis, Djamal Berbiche, François Champagne, Nicole Leduc, Wahid Melki, Khalid Saeed and Fatma Charfi

\section{Background}

Training based on the Mental Health Gap Action Programme (mhGAP) is being increasingly adopted by countries to enhance non-specialists' mental health capacities. However, the influence of these enhanced capacities on referral rates to specialised mental health services remains unknown.

\begin{abstract}
Aims
We rely on findings from a longitudinal pilot trial to assess the influence of mental health knowledge, attitudes and self-efficacy on self-reported referrals from primary to specialised mental health services before, immediately after and 18 months after primary care physicians (PCPS) participated in an mhGAP-based training in the Greater Tunis area of Tunisia.
\end{abstract}

\section{Method}

Participants included PCPs who completed questionnaires before $(n=112)$, immediately after $(n=88)$ and 18 months after $(n=59)$ training. Multivariable analyses with linear mixed models accounting for the correlation among participants were performed with the SAS version 9.4 PROC MIXED procedure. The significance level was $\alpha<0.05$

\section{Results}

Data show a significant interaction between time and menta health attitudes on referrals to specialised mental health services per week. Higher scores on the attitude scale were associated with more referrals to specialised services before and 18 months after training, compared with immediately after training

\section{Conclusion}

Findings indicate that, in parallel to mental health training, considering structural/organisational supports to bring about a sustainable change in the influence of PCPs' mental health attitudes on referrals is important. Our results will inform the scaleup of an initiative to further integrate mental health into primary care settings across Tunisia, and potentially other countries with similar profiles interested in further developing task-sharing initiatives.

\section{Keywords}

Primary care; Tunisia; education and training; outcome studies

\section{Copyright and usage}

(C) The Author(s) 2020. Published by Cambridge University Press on behalf of The Royal college of Psychiatrists. This is an Open Access article, distributed under the terms of the Creative Commons Attribution licence (http://creativecommons.org/ licenses/by/4.0/), which permits unrestricted re-use, distribution, and reproduction in any medium, provided the original work is properly cited.

\section{The Mental Health Gap Action Programme training}

The Mental Health Gap Action Programme (mhGAP) was developed by the World Health Organization (WHO) to bridge the mental health treatment gap (i.e. the gap between the need for mental health services and their delivery ${ }^{1,2}$ ) by developing technical guidance and tools to support decision makers, programme managers and healthcare organisations. ${ }^{3}$ The programme was specifically designed for low- and middle-income countries (LMICs) where the treatment gap is estimated at $76-85 \%,{ }^{2}$ given financial, infrastructure and human resource challenges that influence access to timely mental healthcare. ${ }^{4}$ One of the mhGAP tools is the Intervention Guide (mhGAP-IG), evidence-based guidance to train and support non-specialists in better detecting, treating and managing what the $\mathrm{WHO}$ considers priority mental, neurological and substance use disorders (MNS).

In 2016, a mental health training programme based on the mhGAP-IG was offered to primary care physicians (PCPs) working in the Greater Tunis area of Tunisia, a lower-middleincome country in North Africa. The implementation and evaluation of the training programme based on the mhGAP-IG (version 1.0$)^{5}$ in Tunisia was a collaborative effort between members of the Ministry of Health in Tunisia (particularly the President of the Committee for Mental Health Promotion and the Coordinator of the Technical Committee for Suicide
Prevention), the School of Public Health at Université de Montréal (Canada) and the WHO office in Tunisia. ${ }^{6}$

\section{Factors influencing referral rates}

Although the mhGAP-based training has shown effectiveness in improving non-specialists' mental health capacities to detect and manage MNS disorders, ${ }^{7}$ to our knowledge, there are no studies that assess the influence of mental health capacity (i.e. mental health knowledge, attitudes, confidence in capabilities to detect and manage MNS disorders) acquired through mhGAP-based training on non-specialists' referral rates to specialised mental health services.

A range of factors could be associated with non-specialists' referral rates to specialised mental healthcare. For example, studies show that confidence in capacities to treat mental health conditions ${ }^{8-10}$ and knowledge about these conditions, ${ }^{11}$ as well as attitudes toward specific mental health problems (i.e. what non-specialists may consider to be complex conditions like psychosis and symptoms related to suicidality) or types of patients (i.e. those non-responsive to prescribed medications) are strong predictors of referrals from primary to specialised mental healthcare. ${ }^{9}$ 


\section{Study aim}

The present study aims to improve understanding of the influence of mental health knowledge, attitudes and self-efficacy on selfreported referrals by PCPs to specialised mental health services at three time points: before, immediately after and 18 months after PCPs' participation in the mhGAP-based mental health training programme offered in the Greater Tunis area of Tunisia. Understanding how these capacities influence non-specialists' referrals to specialised mental health services at these three time points will fill a gap in the literature. Findings will provide evidence that may help improve the mhGAP-based training programme design to further foster capacity-building in non-specialised settings; inform the scale-up of a task-sharing programme in Tunisia, centred on further integrating mental health in primary care settings by increasing non-specialists' involvement in mental healthcare delivery and support by specialists; and be relevant to other countries with similar primary care realities and interested in further integrating mental health in primary care settings through, for example, task-sharing initiatives.

\section{Method}

\section{Study design}

This study relies on data collected for the pilot evaluation of the mhGAP-based training in the Greater Tunis area of Tunisia. The pilot study design has been described in detail elsewhere. ${ }^{6}$ In brief, we conducted a pilot trial between January 2016 and September 2017 to assess the effect of the training on PCPs' mental health knowledge, attitudes, self-efficacy and self-reported practice, using several designs. First, a pre-test-post-test control group design $^{12}$ was employed to assess the training's short-term effects (circles 1-4, Fig. 1). In this design, PCPs were randomly assigned to two groups: the intervention group (group 1) and the control group (group 2). Group 1 received the mhGAP-based training between February and March 2016 (circles 1 and 2, Fig. 1), and group 2 received the training between March and April 2016 (circles 4 and 5, Fig. 1). Second, a repeated measures design was employed to assess the training's long-term effects.
For this study, we employed a repeated measures design to further assess data from our pilot evaluation of the mhGAP-based training. ${ }^{6}$ Specifically, we assessed the influence of mental health knowledge, attitudes and self-efficacy on self-reported referrals to specialised mental health services over three time periods, as illustrated in Fig. 1: before the training (circles 1 and 3), immediately after the training of both groups (circles 2 and 5) and 18 months after the training of both groups (circles 6 and 7).

\section{Study setting}

The evaluation of the mhGAP-based training on PCPs' mental health capacities was conducted in the Greater Tunis area of Tunisia, located in the north of the country. ${ }^{6}$ This area includes four governorates: Ariana, Ben Arous, Manouba and Tunis. The Greater Tunis area was chosen as the pilot setting for the first implementation of the mhGAP-based training because it is diverse: it includes governorates that are rural, urban, semi-rural and semiurban, which reflects Tunisia as a whole.

The authors assert that all procedures contributing to this work comply with the ethical standards of the relevant national and institutional committees on human experimentation and with the Helsinki Declaration of 1975 , as revised in 2008. All procedures involving human subjects/patients were approved by the ethics committees of Université de Montréal (Québec, Canada) (approval number \#15-117-CERES-D) and Hôpital Razi (Tunisia).

During the recruitment phase, PCPs were presented the training programme and the study. They were informed that the training and the study were voluntary, and that their decisions related to participation in the training and study would not have any adverse consequences on their employment. Informed consent was obtained from all study participants.

\section{Participants}

Participant recruitment for the pilot trial in which this study is inscribed has been described in detail elsewhere. ${ }^{6}$ We compiled the names of 345 PCPs who were registered with the PCPs' professional order in Tunisia, worked in the public and primary care sectors and previously attended continuing medical education

\begin{tabular}{ll}
$\begin{array}{l}\text { Baseline collection } \\
\text { (January 2016) }\end{array}$ & $\begin{array}{l}\text { Collection post-training, group } 1 \\
\text { (March 2016) }\end{array}$ \\
\hline
\end{tabular}

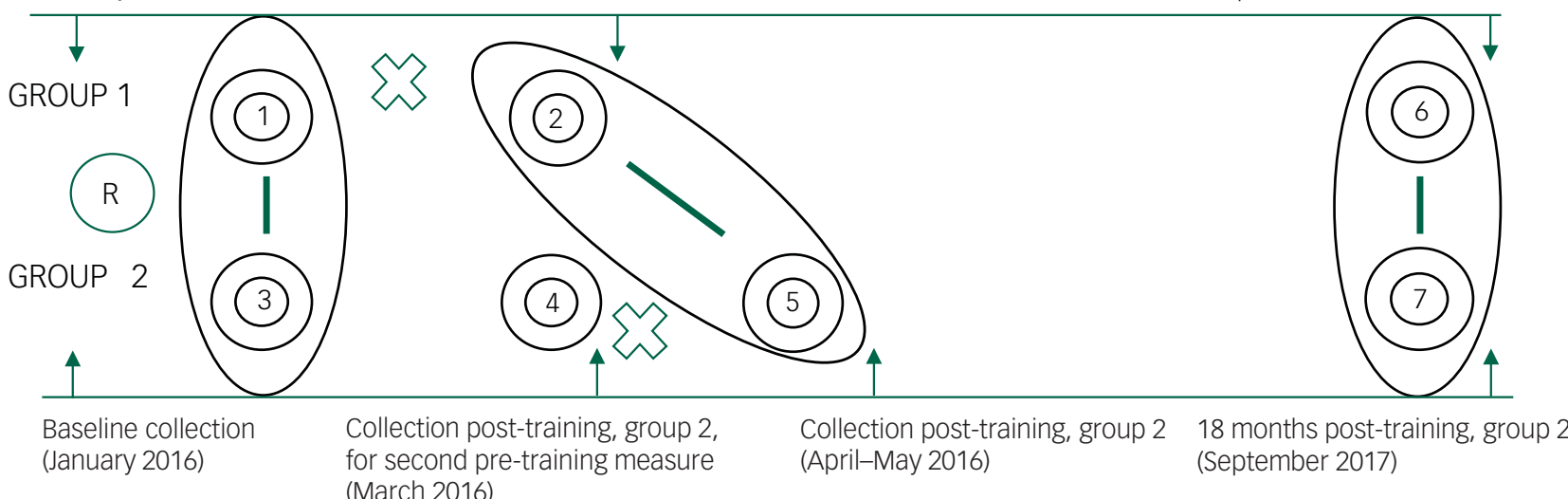

18 months post-training, group 1 (September 2017)

Fig. 1 The design of the pilot trial in which this study is inscribed.

Our study is inscribed within a pilot trial that aimed to assess the mental health capacities of primary care physicians (PCPS) after their participation in Mental Health Gap Action Programme (mhGAP)-based training. For the purposes of this study, we rely on a repeated measures design (as shown in Fig. 1) to improve understanding of the influence of mental health knowledge, attitudes and self-efficacy on self-reported referrals to specialised mental health services at three time periods: pre-training (circles 1 and 3), immediately posttraining (circles 2 and 5) and 18 months post-training (circles 6 and 7). X represents the implementation of the training. R represents when PCPs were randomized to either group 1 or group 2 . The circled areas depict the pooling of group 1 and group 2 over three time periods. This study relies on the secondary data analysis of data collected during the pilot evaluation of the mhGAP-based training in the Greater Tunis area of Tunisia. The pilot study design in which this study is inscribed has been described in detail elsewhere. ${ }^{6}$ 
training. Continuing medical education is recommended and encouraged in Tunisia for advancing PCPs' careers. Of the 345 PCPs, the majority $(91.30 \%)$ worked in primary care in the Greater Tunis area and had 5 or more years of clinical experience, and so were eligible for the study. A total of 132 PCPs (41.90\%) agreed to participate in the pilot trial. In January 2016, the first author contacted the 132 PCPs who agreed to participate in the trial, to further explain the study and to obtain consent. A total of $112(n=112)$ PCPs completed baseline questionnaires and were thus included in the trial.

Forty-five PCPs from group 1 and 43 PCPs from group 2 completed the training programme and agreed to complete questionnaires. This resulted in a pooled total of 88 PCPs included in the analyses immediately after the training. Thirty-two PCPs from group 1 and 27 PCPs from group 2 agreed to complete questionnaires at 18 months (September 2017) post-training. This resulted in a pooled total of 59 PCPs included in long-term analysis.

\section{Intervention}

The mhGAP-based training (version 1.0) ) $^{5}$ was offered to PCPs in the Greater Tunis area between February and May 2016. The programme's adaptation to the local context has been described elsewhere. ${ }^{13}$ Members of the Ministry of Health in Tunisia (W.M., F. Charfi) chose specific training modules considered important to Tunisia: general principles of care, depression, psychosis, suicide/ self-harm and problems related to substance use. These modules were adapted to meet the Greater Tunis area's primary care realities, with the help of W.M., F. Charfi, three Tunisian psychiatrists ('trainers') and seven PCPs responsible for continuing medical education in the Greater Tunis area who had the role of supporting trainees during and post-training ('tutors'). The training lasted 6 weeks for a total of $19 \mathrm{~h}$, with sessions being offered once a week. The first five sessions comprised general lectures, role-plays and group discussions $(17 \mathrm{~h})$. The last session was a 2-h support session offered by the trainers consisting of further role-plays to help PCPs with challenging mental health cases.

\section{Measures}

Variables for the pilot trial ${ }^{6}$ were chosen according to Kirkpatrick's conceptual model, ${ }^{14}$ often used for training programme evaluations. ${ }^{15}$

\section{Independent variables}

Mental health knowledge was assessed using the questionnaire developed by the WHO to accompany the mhGAP training programme. The questionnaire contained 16 questions on the modules selected for training. Correct answers were scored as 1 and incorrect answers were scored as 0. A participant's score is the sum of correct answers for individual items. Overall knowledge scores were converted to a score ranging from 0 to 10 , where a higher score indicates more knowledge. This questionnaire reported a good degree of reliability in our sample (the average measure Intraclass Correlation Coefficient (ICC) was 0.708 , with a $95 \%$ confidence interval of $0.478-0.837) .{ }^{16}$ These psychometric analyses have been reported elsewhere. ${ }^{16}$

The Mental Illness: Clinicians' Attitudes (MICA) Scale (version 4.0) was used to measure attitudes toward mental illness and the field of mental health. ${ }^{17-18}$ The scale's Cronbach's alpha, tested on our sample, was found to be poor (0.521). To increase the scale's internal consistency, we kept 11 items (i.e. questions $1,2,4,5,7$, $10,12,13,14,15$ and 16) from the MICA-4 (version 4.0), which resulted in an increase in the total Cronbach's alpha (0.608). Cronbach's alpha is a function of scale length, and increased in our case by removing five items from the original scale. Hence, the new Cronbach's alpha was sufficient. ${ }^{16}$ This procedure has been explained in detail elsewhere. ${ }^{16}$ For statements 10,12 and 16 , items were scored on a scale of 1-6, from 'strongly agree' to 'agree', 'somewhat agree', 'somewhat disagree', 'disagree' and 'strongly disagree'. All other items were reverse scored. Scores on individual items were summed to obtain each participant's overall score within a range of 11-66 points. A higher global score indicates a more negative perception about MNS disorders and mental healthcare delivery. This modified questionnaire reported a good degree of reliability in our sample (the average measure of the ICC was 0.704 , with a $95 \%$ confidence interval of $0.468-0.835) .{ }^{16}$

The self-efficacy questionnaire was developed for the pilot trial and comprised 35 questions about PCPs' judgement of their capability to detect, treat and manage mental health conditions included in the training programme. The scale's Cronbach's alpha, tested on our sample, was found to be satisfactory (0.937). Each statement was scored on a scale of 0-4, from 'strongly agree' to 'somewhat agree', 'neutral', 'somewhat disagree' and 'strongly disagree'. A participant's overall score is the sum of answers for individual items. Overall scores were converted to a score ranging from 0 to 10 , where a higher score indicates more self-efficacy. This questionnaire has shown a good degree of reliability in our sample (the average measure of the ICC was 0.781 , with a $95 \%$ confidence interval of $0.606-0.878){ }^{16}$ These psychometric analyses have been reported elsewhere. ${ }^{16}$

\section{Dependent variable}

Our variable of interest was PCPs' self-reported referrals to specialised mental health services, which represents the average weekly percentage of mental health clientele that PCPs reported referring to specialised mental health services. Specifically, PCPs were asked the following question related to their self-reported referrals: among patients presenting with mental health problems, what percentage do you refer to specialised mental health services per week? The variable 'self-reported referrals to specialised mental health services' is evaluated on a score that ranges between 0 and $100 \%$.

\section{Covariates}

Baseline sociodemographic (age, gender) and practice characteristics, such as part-time or full-time work, average number of weekly work hours, average number of weekly hours dedicated to mental health, average number of weekly patient consultations, average number of weekly patient consultations specifically for mental health, average weekly percentage of consultations per type of mental health condition and previous mental health training, were examined as potential covariates.

\section{Data collection}

All questionnaires were pre-tested ${ }^{16}$ and administered at four time points: at baseline, before randomisation (January 2016); following group 1's training (March 2016); following group 2's training (April and May 2016); and 18 months after the training (September 2017) (Fig. 1).

\section{Analyses}

This study is a secondary analysis of data collected within the context of a pilot trial. ${ }^{6}$ To assess whether PCPs' mental health knowledge, attitudes and self-efficacy predict PCPs' self-reported referrals to specialised mental health services, we conducted multiple linear regression analyses. Multivariable analyses with linear mixed models, accounting for the correlation among participants, were performed with the SAS version 9.4 PROC MIXED procedure (platform X64_8PRO for Windows). One of the strengths of these 
models is that they consider an unequal number of measurements per participant (owing to attrition and/or non-response) for a given time period and even if time intervals are not constant. The level of significance was set at $\alpha<0.05$.

\section{Results}

Table 1 summarises the sociodemographic and practice characteristics of PCPs who completed questionnaires before participation in the training $(n=112)$, after PCPs assigned to groups 1 and 2 participated in the training $(n=88)$ and 18 months after training $(n=59)$.

Most PCPs included in our sample were women. At baseline, participants had a median of 49 years of age. Few PCPs reported having any mental health training in the 12 months before the implementation of the mhGAP-based training. At baseline, PCPs estimated that they saw approximately 139 patients per week, with a median of 12 patients consulting for mental health problems. Of their 36-h work week, PCPs reported dedicating $<4 \mathrm{~h}$ to mental health. PCPs self-reported providing consultation for anxiety and depression, primarily.

Before training, PCPs scored lower on the knowledge questionnaire, higher on the attitude questionnaire, lower on the self-efficacy questionnaire and reported more referrals to specialised mental health services than immediately and 18 months after the training. ${ }^{6}$ No differences in characteristics were found between completers and non-completers at 18 months after training. Some differences were found between completers and non-completers immediately after training. These include scores on the attitude questionnaire $(P=0.016)$ and self-reported percentages of consultations for problems related to alcohol use $(P=0.039)$ and drug use $(P=0.037)$. However, these differences remain minimal.

The multivariable analyses presented in Table 2 shows some general trends, like statistically significant associations between PCPs' self-reported percentage of referrals to specialised mental health services per week and several baseline characteristics across all time points. Specifically, PCPs with less experience (i.e. who were younger) reported higher levels of referrals; PCPs who reported a higher percentage of patients consulting for problems related to psychosis per week also reported higher levels of

\begin{tabular}{|c|c|c|c|c|c|}
\hline \multirow[b]{2}{*}{ Sociodemographic characteristics } & \multicolumn{5}{|c|}{ Time of data collection } \\
\hline & Before $(n=112)$ & $\begin{array}{l}\text { Immediately after } \\
\qquad(n=88)\end{array}$ & $P$ value $^{a}$ & $\begin{array}{l}18 \text { months after } \\
\qquad(n=59)\end{array}$ & $P$ value $^{\mathrm{b}}$ \\
\hline Age, years, median (Q1, Q3) & $49.00(46.00,53.00)$ & $48.00(45.00,52.00)$ & 0.209 & $48.00(45.00,52.00)$ & 0.121 \\
\hline \multicolumn{6}{|l|}{ Gender, $n(\%)$} \\
\hline Women & $90(80.40)$ & $73(83.00)$ & \multirow{2}{*}{0.244} & $50(84.70)$ & \multirow{2}{*}{0.523} \\
\hline Men & $22(19.60)$ & $15(17.00)$ & & $9(15.30)$ & \\
\hline \multicolumn{6}{|l|}{ Practice characteristics } \\
\hline \multicolumn{6}{|l|}{ Governorate, $n$ (\%) } \\
\hline Tunis & $43(38.40)$ & $33(37.50)$ & \multirow[t]{4}{*}{0.341} & $22(37.30)$ & \multirow[t]{4}{*}{0.519} \\
\hline Manouba & $21(18.80)$ & $16(18.20)$ & & $10(16.90)$ & \\
\hline Ben Arous & $20(17.90)$ & $14(15.90)$ & & $9(15.30)$ & \\
\hline Ariana & $28(25.00)$ & $25(28.40)$ & & $18(30.50)$ & \\
\hline \multicolumn{6}{|l|}{ Work, $n(\%)$} \\
\hline Part time & $28(25.00)$ & $19(21.60)$ & \multirow[t]{2}{*}{0.110} & $10(16.90)$ & \multirow[t]{2}{*}{0.131} \\
\hline Full time & $84(75.00)$ & $69(78.40)$ & & $49(83.10)$ & \\
\hline Hours of work per week, median (Q1, Q3) & $36.00(30.00,36.00)$ & $36.00(34.00,36.00)$ & 0.693 & $36.00(36.00,36.00)$ & 0.193 \\
\hline $\begin{array}{l}\text { Average number of hours dedicated to mental healthcare } \\
\text { per week, median }(\mathrm{Q} 1, \mathrm{Q} 3)\end{array}$ & $3.60(2.10,6.00)^{c}$ & $3.60(2.16,7.20)$ & 0.886 & $3.60(1.08,7.20)$ & 0.957 \\
\hline $\begin{array}{l}\text { Average number of patient consultations per week, } \\
\text { median }(\mathrm{Q} 1, \mathrm{Q} 3)\end{array}$ & $138.50(103.75,180.00)$ & $125.00(100.00,180.00)$ & 0.845 & $140.00(100.00,180.00)$ & 0.385 \\
\hline $\begin{array}{l}\text { Average number of patient consultations for mental } \\
\text { health per week, median (Q1, Q3) }\end{array}$ & $12.00(4.95,21.06)$ & $4.80(2.10,15.00)$ & 0.241 & $8.75(2.85,19.18)$ & 0.439 \\
\hline \multicolumn{6}{|c|}{ Percentage of mental health consultations per week according to diagnosis } \\
\hline \multicolumn{6}{|c|}{ Types of mental health consultation per week, median (Q1, Q3) } \\
\hline Anxiety & $50.00(30.00,70.00)$ & $30.00(20.00,60.00)$ & 0.055 & $50.00(25.00,70.00)$ & 0.184 \\
\hline Depression & $30.00(20.00,45.00)$ & $30.00(10.00,50.00)$ & 0.467 & $30.00(20.00,50.00)$ & 0.077 \\
\hline Alcohol use disorders & $3.00(0.00,10.00)$ & $2.00(0.00,10.00)$ & $0.039 *$ & $2.00(1.00,10.00)$ & 0.352 \\
\hline Drug use disorders & $2.00(0.00,10.00)$ & $1.00(0.00,5.00)$ & $0.037^{\star}$ & $1.00(0.00,5.00)$ & 0.553 \\
\hline Psychosis (including schizophrenia) & $2.00(0.00,9.00)$ & $2.00(0.00,10.00)$ & 0.691 & $3.00(0.04,10.00)$ & 0.074 \\
\hline Suicide/self-harm & $1.00(0.00,5.00)$ & $1.00(0.00,2.00)$ & 0.254 & $3.00(0.00,3.00)$ & 0.360 \\
\hline \multicolumn{6}{|c|}{ Mental health training before intervention (Jan 2015 to Jan 2016), $n$ (\%) } \\
\hline Yes & $14(12.50)$ & $12(13.60)$ & \multirow[t]{2}{*}{0.486} & $8(13.60)$ & \multirow[t]{2}{*}{0.976} \\
\hline No & $98(87.50)$ & $76(86.40)$ & & $51(86.40)$ & \\
\hline \multicolumn{6}{|l|}{ PCPs' mental health capacities } \\
\hline Knowledge about mental health, median (Q1, Q3) & $6.25(5.63,7.50)$ & $7.50(6.88,8.75)$ & 0.684 & $7.50(6.25,8.13)$ & 0.147 \\
\hline Attitudes about mental health, median (Q1, Q3) ${ }^{\mathrm{d}}$ & $28.00(24.00,32.00)$ & $25.00(20.00,28.00)$ & $0.016^{*}$ & $27.00(20.00,32.00)$ & 0.942 \\
\hline Self-efficacy in mental healthcare, median (Q1, Q3) & $5.21(4.08,6.28)$ & $7.32(6.36,8.05)$ & 0.552 & $6.14(5.29,7.29)$ & 0.796 \\
\hline PCPS' referral habits, median (Q1, Q3) & $50.00(30.00,80.00)^{c}$ & $30.00(8.50,60.00)$ & 0.818 & $40.00(10.00,70.00)^{c}$ & 0.445 \\
\hline \multicolumn{6}{|c|}{$\begin{array}{l}\text { a. The } P \text {-value describes the differences in characteristics between the completers (study participants who completed the questionnaires immediately after the training) and the non- } \\
\text { completers (study participants who did not complete the questionnaires immediately after the training) compared with pre-training. Independent } t \text {-tests for continuous variables and } \chi^{2} \text { tests } \\
\text { for categorical variables were performed. } \\
\text { b. The } P \text {-value describes the differences in characteristics between the completers (study participants who completed the questionnaires at } 18 \text { months post-training) and the non-com- } \\
\text { pleters (study participants who did not complete the questionnaires at } 18 \text { months after the training) compared with immediately after the training. Independent } t \text {-tests for continuous } \\
\text { variables and } \chi^{2} \text { tests for categorical variables were performed. } \\
c \text {. Missing values were }>5 \% \text { but }<10 \% \text {. } \\
\text { d. This scale is reverse scored (a higher score indicates more negative attitudes toward mental health and illness). } \\
* P<0.05 \text {. }\end{array}$} \\
\hline
\end{tabular}




\begin{tabular}{|c|c|c|c|}
\hline Characteristics & Estimates & Confidence intervals & $P$ value \\
\hline \multicolumn{4}{|l|}{ Time } \\
\hline Baseline & 11.942 & $(1.996-21.888)$ & $0.019^{*}$ \\
\hline Immediately after training & 0 & - & - \\
\hline 18 months after training & -0.179 & $(-9.674$ to 9.315$)$ & 0.970 \\
\hline \multicolumn{4}{|l|}{ Group } \\
\hline 1 & 7.643 & $(-1.064$ to 16.351$)$ & 0.084 \\
\hline 2 & 0 & - & - \\
\hline \multicolumn{4}{|l|}{ Mental health capacities } \\
\hline Knowledge about mental health & -6.330 & $(-9.347$ to -3.314$)$ & $<0.000^{*}$ \\
\hline Attitudes about mental health & -0.301 & $(-0.967$ to 0.364$)$ & 0.371 \\
\hline Self-efficacy in mental healthcare & -0.379 & (-3.249 to 2.490$)$ & 0.793 \\
\hline \multicolumn{4}{|l|}{ Sociodemographic characteristics } \\
\hline \multirow{2}{*}{\multicolumn{4}{|c|}{ Gender }} \\
\hline & & & \\
\hline Female & 7.466 & $(-4.148$ to 19.081$)$ & 0.205 \\
\hline Male & 0 & - & - \\
\hline \multicolumn{4}{|l|}{ Practice characteristics } \\
\hline \multicolumn{4}{|l|}{ Work } \\
\hline Part time & 6.371 & $(-8.836$ to 21.579$)$ & 0.407 \\
\hline Full time & 0 & - & - \\
\hline \multicolumn{4}{|l|}{ Previous mental health training (Jan 2015 to Jan 2016) } \\
\hline No & 8.774 & $(-4.041$ to 21.589$)$ & 0.177 \\
\hline Yes & 0 & - & - \\
\hline Hours of work per week & 0.423 & $(-0.938$ to 1.785$)$ & 0.538 \\
\hline Average number of hours dedicated to mental healthcare per week & 0.020 & $(-0.340$ to 0.381$)$ & 0.909 \\
\hline Average number of consultations per week & -0.026 & $(-0.098$ to 0.046$)$ & 0.476 \\
\hline Average number of consultations for mental health per week & 0.0889 & $(-0.283$ to 0.461$)$ & 0.636 \\
\hline Percentage of consultations for anxiety per week & 0.150 & $(-0.000$ to 0.302$)$ & 0.051 \\
\hline Percentage of consultations for depression per week & 0.081 & (-0.089 to 0.253$)$ & 0.344 \\
\hline Percentage of consultations for alcohol use per week & 0.214 & $(-0.172$ to 0.602$)$ & 0.274 \\
\hline Percentage of consultations for drug use per week & -0.253 & $(-0.608$ to 0.101$)$ & 0.160 \\
\hline Percentage of consultations for psychosis per week & 0.385 & $(0.060-0.710)$ & $0.020^{*}$ \\
\hline Percentage of consultations for suicide/self-harm per week & -0.084 & $(-0.460$ to 0.291$)$ & 0.658 \\
\hline
\end{tabular}

referrals; PCPs who scored higher on the mental health knowledge questionnaire reported fewer referrals to specialised mental health services.

The multivariable analyses testing for the presence of an interaction between time and PCP study factors on referrals (Table 3) showed a significant interaction between time and attitudes about mental health on referrals to specialised mental health services per week. Higher levels of negative attitudes were significantly associated with more self-reported referrals to specialised mental health services before and at 18 months after the training programme, compared with immediately after the training. No significant interaction between time and PCPs' knowledge and selfefficacy about mental health on referrals to specialised mental health services was found.

\section{Discussion}

This study aimed to improve understanding of the influence of PCPs' mental health knowledge, attitudes and self-efficacy on their self-reported referrals to specialised mental health services before, immediately after and 18 months after the implementation of an mhGAP-based training. The general trend seemed to be that scores on mental health knowledge and self-efficacy questionnaires influenced PCPs' self-reported percentage of referrals to specialised mental health services similarly across the three time points. However, we found a significant interaction between time and PCPs' level of attitudes on referrals to specialised mental health services per week. We contextualise our findings within the literature

\begin{tabular}{|c|c|c|c|}
\hline Mental health capacities $\times$ time & Estimates & Confidence intervals & $P$ value \\
\hline Knowledge about mental health $\times$ time & & & 0.156 \\
\hline Baseline & -4.357 & $(-10.190$ to 1.474$)$ & 0.141 \\
\hline Immediately after training & 0 & - & - \\
\hline 18 months after training & 1.828 & $(-5.596$ to 9.251$)$ & 0.626 \\
\hline Attitudes about mental health $\times$ time & & & $0.009 *$ \\
\hline Baseline & 1.612 & $(0.330-2.895)$ & $0.014^{*}$ \\
\hline Immediately after training & 0 & - & - \\
\hline 18 months after training & 2.066 & $(0.5970-3.5356)$ & $0.006^{*}$ \\
\hline Self-efficacy in mental healthcare $\times$ time & & & 0.978 \\
\hline Baseline & 0.512 & $(-5.055$ to 6.081$)$ & 0.855 \\
\hline Immediately after training & 0 & - & - \\
\hline 18 months after training & -0.000 & $(-6.958$ to 6.956$)$ & 0.999 \\
\hline
\end{tabular}


on the theory of planned behaviour, where 'attitudes, subjective norms and perceived behavioral control are shown to be related to appropriate sets of salient behavioral, normative, and control beliefs about the behaviour'. ${ }^{19}$

Our findings seem to highlight that in the backdrop of a mental health training programme, attitudes toward MNS and mental healthcare delivery may, over time, influence behaviours such as referrals to specialised mental health services more so than capacities like knowledge and self-efficacy, which we found to have no time interaction with reported referrals. Specifically, we found that higher scores on the attitude scale were associated with more referrals to specialised services before and 18 months after training, compared with immediately after training. Hence, the mhGAP training may not be enough over the longer term. Mental health stigma is still widespread in Tunisia, the Arab world and more generally in LMICs. This stigma continues to be translated into practice. For example, studies show that it is common for healthcare professionals to believe that people with mental illness are violent and dangerous, that their condition is a personal or moral fault and that treatment by mental health specialists is preferred. ${ }^{16,20-24}$ This reality might also be reflected in Tunisia: most consultations for mental healthcare continue to be provided in specialised services, ${ }^{25}$ despite the uneven distribution of mental health specialists across the country ${ }^{25}$ and the ministerial vision of further integrating mental health into primary care settings. ${ }^{26}$ Mental health stigma may be instilled in social norms and may help explain, in part, PCPs' choice in referring to specialists versus delivering mental healthcare over the longer term despite the implementation of a mental health training.

Furthermore, the broader context consisting of public health policies and regulations may influence PCPs' attitudes toward mental healthcare delivery and their choice in referring to specialised mental healthcare. For example, PCPs in Tunisia cannot prescribe certain medications used to treat mental health conditions; ${ }^{27}$ and laws before the Tunisian Revolution often considered people with problems related to substance misuse as social offenders, which may have shaped many PCPs' 'fear' of these types of conditions. ${ }^{27}$ Organisational realities might also increase negative perceptions of mental healthcare delivery and thus referrals to specialised mental healthcare in Tunisia. For example, PCPs expressed lacking organisational support to motivate mental healthcare delivery (i.e. staff meetings to discuss challenging mental health cases, access to psychotropic medications). ${ }^{27}$ This larger context is often overlooked in the theory of planned behaviour. However, it might have had an effect on past experiences, which, in turn, can challenge anticipated behaviours especially over the longer term when the training's infrastructure and support may no longer be available.

Solutions to address these contextual issues should not only focus on skills-based training (like the mhGAP-based programme we offered ${ }^{6}$ ), but also on modifying policies and regulations that may help to ensure that mental health is included within universal healthcare packages and in developmental assistance plans across sectors. This inclusion may ultimately have a positive effect on mental health attitudes and thus on referrals to specialised mental healthcare. Specifically, mental health training programmes offered to non-specialists like PCPs might help to lessen their negative mental health attitudes, which can consequently effect their referrals to specialised mental health services per week immediately after the training, as shown by our current study. But, organisational supports and policies (regular supervision from specialists, the availability of psychotropic medications, changes in regulations to allow PCPs to prescribe essential psychotropic medications) are also needed to bring about a sustainable change in the attitudes of PCPs toward mental health to help maintain positive practice effects, ${ }^{6,27}$ including self-reported referrals. Studies also highlight the roles that healthcare organisations can play in tackling mental health stigma and in further encouraging non-specialists in mental healthcare delivery. These include strong leadership support, monitoring mechanisms related to quality of care, and opportunities for non-specialists to engage in social contact with people with lived experience who are trained in sharing their recovery journeys and experiences in navigating the healthcare system. ${ }^{28-}$

31 These broader changes in mental health policy and legislation occurring in parallel with building non-specialists' mental health capacities and implementing organisational supports are essential ingredients to inform mental healthcare delivery in non-specialised settings in Tunisia, and more broadly in LMICs. Interestingly, Tunisia's scale-up of a mental health training across the country, which will be offered to PCPs, will include a longer-term vision of supervision and support by specialists to further build mental health capacity in primary care settings. In addition, legislation on PCPs' prescription of essential psychotropic medications is being revised for scale-up of the training programme. The importance of further integrating mental health into primary care settings in the country has also been acknowledged in recent reforms to the mental health curricula offered to family physicians. It now includes additional mental health courses and a mental health mandatory internship, which was previously optional. ${ }^{32}$ Future studies should include evaluation components related to the effect of these initiatives on attitudes toward mental healthcare delivery and their influence on referrals to specialised mental health services via primary care settings.

In LMICs, mental health specialists are already scarce, unevenly distributed and/or unavailable. ${ }^{4}$ Yet, they are often the 'go to' for mental health consultations. ${ }^{4,25}$ Mental health systems must be ready now and over the longer term to ensure that mental healthcare is offered to the wider population, and especially to those considered more vulnerable to the residual effects of the coronavirus 2019 pandemic. ${ }^{33}$ Hence, task-sharing (i.e. the increased involvement of nonspecialists like PCPs in mental healthcare delivery ${ }^{34-35}$ ) may be a viable option in the context of the pandemic and beyond, should training and longer-term support be offered to non-specialists. ${ }^{33}$ Task-sharing will, however, continue to require changes in the conception of mental healthcare delivery of many LMICs, including Tunisia.

\section{Limitations and strengths}

First, since our measures are based on self-reports, they could have been subject to social desirability bias, especially in the post-training measurements. Therefore, self-reports should be considered approximates. However, given that mental health statistics within clinics are not digitalised in Tunisia and thus pose a challenge in record-keeping and consultation, ${ }^{27}$ self-reporting was a feasible option. In addition, studies show that self-reporting practice characteristics are likely to produce reliable information in the context of measuring outcomes related to training programmes. ${ }^{36}$

Second, we cannot ascertain if the study's results are generalisable to all PCPs working in Tunisia. However, we assume that the influence of mental health capacities on PCPs' self-reported percentage of referrals to specialised mental health services per week might be similar in other areas of Tunisia, should PCPs agree to participate in the training program's scale-up.

Third, two of the scales used (i.e. mental health knowledge and self-efficacy) were not previously validated. However, psychometric properties of these scales were assessed and proved acceptable. ${ }^{16}$

Last, we acknowledge having lost about half of our sample at 18 months post-training. However, the potential selection bias of this loss to follow-up is minimised as there was no statistical difference 
in the participants' sociodemographic characteristics, mental health capacities and self-reported referrals to specialised mental health services between completers and non-completers at 18 months. In other words, study participants who remained in the study at 18 months despite a $50 \%$ attrition rate were similar to non-completers in all variables assessed (Table 1). However, some variables are worth discussing. Completers immediately after the training had statistically significant lower scores on the attitude questionnaires compared with non-completers. Had the non-completers remained in the study, level of attitudes may have been more negative and therefore may have had a greater influence on referrals to specialised mental health services. We notice that some participants (approximately $13 \%$ ) had already participated in mental health training in the year before the mhGAP-based training and study enrolment. The proportion of study participants who participated in a previous mental health training remained consistent immediately and at 18 months after training. Interestingly, we observe the same trend for PCPs who had not participated in mental health training before the mhGAP-based training. For example, approximately $88 \%$ had not previously participated in a mental health training in the year before the mhGAP-based training and study enrolment, and this proportion remained at approximately $86 \%$ immediately and at 18 months after training. Hence, the level of bias related to participant engagement in mental health training is limited. It is worth noting that we lost some participants who worked part time. This finding may be explained by beliefs that mental healthcare delivery could require additional time beyond pre-existing clinical commitments. ${ }^{27}$ We also noticed that PCPs reported more mental health consultations per week before participating in the mental health training. We hypothesize that lower levels immediately and 18 months after training may have been because of more accurate diagnosis owing to the training and/or an overestimation of self-reported mental health consultations before training. Quality indicators for referrals in the context of training programmes like the mhGAP should be explored in future studies.

Jessica Spagnolo D, MSW, PhD, School of Public Health, University of Montreal; Department of Community Health Sciences, University of Sherbrooke; and Charles-LeMoyne - Saguenay-Lac-St-Jean Research Centre on Health Innovations, University of Sherbrooke - Longueuil Campus, Quebec, Canada; Helen-Maria Vasiliadis, MSc, PhD, Department of Community Health Sciences, University of Sherbrooke; and Charles-LeMoyne - Saguenay-Lac-St-Jean Research Centre on Health Innovations, University of Sherbrooke - Longueuil Campus, Quebec, Canada; Djamal Berbiche, PhD, Department Sherbrooke - Longueuil Campus, Quebec, Canada; Djamal Berbiche, PhD, Depart
of Community Health Sciences, University of Sherbrooke; and Charles-Le-Moyne of Community Health Sciences, University of Sherbrooke; and Charles-Le-Moyne -
Saguenay-Lac-St-Jean Research Centre on Health Innovations, University of Sherbrooke Longueuil Campus, Quebec, Canada; François Champagne, PhD, School of Public Health, University of Montreal, Quebec, Canada; Nicole Leduc, PhD, School of Public Health, University of Montreal, Quebec, Canada; Wahid Melki, Department of Psychiatry D, Razi Hospital; Faculty of Medicine of Tunis, University of Tunis El-Manar, Tunisia; and Technical Committee for Mental Health Promotion in Ministry of Health, Tunis, Tunisia; Khalid Saeed, FCPS (Psych), Mental Health and Substance Abuse Unit, Department of Non-Communicable Diseases and Mental Health, World Health Organization Regional Office for the Eastern Mediterranean, Egypt; Fatma Charfi, Department of Child Psychiatry, Mongi Slim Hospital; and Faculty of Medicine of Tunis, University of Tunis El-Manar, Tunisia

Correspondence: Jessica Spagnolo. Email: jessica.spagnolo@usherbrooke.ca

First received 24 Jun 2020, final revision 16 Sep 2020, accepted 24 Sep 2020

\section{Data availability}

The data that support the findings of this study are available from the corresponding author, J.S., upon reasonable request.

\section{Acknowledgements}

The authors thank all study participants. Their participation was instrumental in generating the results presented in this paper. The authors also thank Dr Guido Sabatinelli, former WHO Representative in Tunisia, and Ann-Lise Guisset, PhD, former WHO Health System Advisor in Tunisia, for their feedback on the questionnaires used in this study and technical support while J.S. was in Tunisia; trainer psychiatrists Dr Trabelsi Sonda, Dr Nesrine Bram and Dr Gasmi Imen, and clinicians Dr Bouabid Leila, Dr Ben Hadj Hassine Ganzoui Sana, Dr Saoud
Zeineb, Dr Zine Elhem, Dr Bannour Saida, Dr Ben Mhenni Mongi and Dr Riahi Ali, responsible for continuing medical education in the Greater Tunis area of Tunisia, for their input on the training programme and help with participant recruitment; and the WHO office in Tunisia, for its administrative support while J.S. was in Tunisia. J.S. would like to thank Matthew Rettino for his editing services.

\section{Author contributions}

J.S., F. Champagne, N.L., W.M. and F. Charfi conceived the larger pilot trial in which this study is inscribed. They also chose and/or developed the scales used to collect data for this study. J.S conducted data collection and data entry came up with the idea for this paper and wrote the first draft of the manuscript. H.-M.V. helped conceive data analysis and interpret the data. D.B. conducted data analysis, wrote the data analysis section of the manuscript and helped interpret the data. F. Charfi and W.M. were instrumental in helping to contextualise the findings to Tunisia. K.S. critically analysed the manuscript from the perspective of a WHO mental health expert working in the Eastern Mediterranean Region (the WHO area in which Tunisia is located). All authors revised and approved the final version of the paper.

\section{Funding}

While this study was conducted, J.S. was supported by a research doctoral training grant awarded by Fonds de recherche du Québec - Santé (FRQS) (2016-2018, project \#33774). J.S. is currently supported by a research postdoctoral training grant (FRQS, 2020-2022, project \#284461). Data collection inscribed within this study was supported by a grant from Mitacs Globalink (research fellowship \#T06835). The overall project in which this data collection was conducted was supported by Institut de recherche en santé publique de l'Université de Montréal - Nouvelles Initiatives.

\section{Declaration of interest}

K.S. is employed by the World Health Organization. All other authors declare that they have no conflicts of interest.

ICMJE forms are in the supplementary material, available online at https://doi.org/10.1192/ bjo.2020.115.

\section{References}

1 Patel V, Saxena S, Lund C, Thornicroft G, Baingana F, Bolton P, et al. The Lancet Commission on global mental health and sustainable development. Lancet 2018; 392(10157): 1553-98.

2 World Health Organization (WHO). Mental Health Action Plan, 2013-2020. WHO 2013 (http://apps.who.int/iris/bitstream/10665/89966/1/9789241506021_eng. pdf?ua=1).

3 World Health Organization (WHO). mhGAP Operations Manual. WHO, 2018 (https://apps.who.int/iris/bitstream/handle/10665/275386/9789241514811eng.pdf?ua=1).

4 World Health Organization (WHO). Mental Health Atlas 2017. WHO, 2018 (http:// apps.who.int/iris/bitstream/handle/10665/272735/9789241514019-eng.pdf? $\mathrm{ua}=1$ ).

5 World Health Organization (WHO), mhGAP Intervention Guide for Mental, Neurological and Substance Use Disorders in Non-Specialized Health Settings (Version 1.0). WHO, 2010 (http://apps.who.int/iris/bitstream/handle/10665/ 44406/9789241548069 eng.pdf;jsessionid=A1FF1B6443F6185A511855E97BB 665F8? sequence=1).

6 Spagnolo J, Champagne F, Leduc N, Rivard M, Melki W, Piat M, et al. Building capacity in mental health care in low- and middle-income countries by training primary care physicians using the mhGAP: a randomized controlled trial. Health Policy Plan 2020; 35(2): 186-98.

7 Keynejad RC, Dua T, Barbui C, Thornicroft G. WHO Mental Health Gap Action Programme (mhGAP) Intervention Guide: a systematic review of evidence from low and middle-income countries. Evid Based Ment Health 2018; 21(1): 30-4.

8 Anthony JS, Baik S, Bowers BJ, Tidjani B, Jacobson CJ, Susman J. Conditions that influence a primary care clinician's decision to refer patients for depression care. Rehabil Nurs 2010; 35(3): 113-22.

9 Steele M, Zayed R, Davidson B, Stretch N, Nadeau L, Fleisher W, et al. Referral patterns and training needs in psychiatry among primary care physicians in Canadian rural/remote areas. J Can Acad Child Adolesc Psychiatry 2012; 21 (2): 111-23.

10 Tzartzas K, Oberhauser PN, Mario-Veyron R, Bourquin C, Senn N, Stiefel F. General practitioners referring patients to specialists in tertiary healthcare: a qualitative study. BMC Fam Med 2019; 20: 165

11 Ringberg U, Fleten N, Helge Førde O. Examining the variation in GPs' referral practice: a cross-sectional study of GPs' reasons for referral $\mathrm{Br} J$ Gen Pract 2014; 64(624): e426-33.

12 Campbell DT, Stanley JC, Gage NL. Experimental and Quasi-Experimental Designs for Research. Houghton Mifflin, 1963.

13 Spagnolo J, Champagne F, Leduc N, Melki W, Guesmi I, Bram N, et al. Tailoring a training based on the Mental Health Gap Action Programme (mhGAP) 
Intervention Guide (IG) to Tunisia: process and relevant adaptations. Glob Ment Health (Camb) 2018; 5: e17.

14 Kirkpatrick DL, Kirkpatrick JD. Evaluating Training Programs: The Four Levels (3rd edn). Berrett-Koehler, 2006

15 Smidt A, Balandin S, Sigafoos J, Reed VA et al. The Kirkpatrick model: a useful too for evaluating training outcomes. J Intellect Dev Disabil 2009; 34(3): 266-74.

16 Spagnolo J, Champagne F, Leduc N, Rivard M, Piat M, Laporta M, et al. Mental health knowledge, attitudes, and self-efficacy among primary care physicians working in the Greater Tunis area of Tunisia. Int J Ment Health Syst 2018; 26 (12): 63

17 Gabbidon J, Clement S, van Nieuwenhuizen A, Kassam A, Brohan E, Norman I, et al. Mental Illness: Clinicians' Attitudes (MICA) scale-psychometric propertie of a version for healthcare students and professionals. Psychiatry Res 2013 206(1): 81-7.

18 INDIGO. Stigma Scales. The INDIGO Network, 2018 (http://www.indigogroup. org/stigma-scales/).

19 Ajzen I. The theory of planned behavior. Organ Behav Hum Decis Process 1991; 50: $172-211$.

20 Al Saif F, Al Shakhoori H, Nooh S, Jahrami H. Association between attitudes of stigma toward mental illness and attitudes toward adoption of evidence-based practice within health care providers in Bahrain. PLOS ONE 2019; 14(2): e0025738.

21 Haddad M, Waqas A, Qayyum W, Shams M, Malik S. The attitudes and beliefs of Pakistani medical practitioners about depression: a cross-sectional study in Lahore using the revised depression attitude questionnaires (R-DAQ). BMC Psychiatry 2016; 16(1): 349

22 Henderson C, Evans-Lacko S, Thornicroft G. Mental illness stigma, help seeking and public health programs. Am J Public Health 2013; 103(5): 777-80.

23 Ola B, Crabb J, Adewuya A, Olugbile F, Abosede OA. The state of readiness of Lagos State primary health care physicians to embrace the care of depression in Nigeria. Community Ment Health J 2014; 50(2): 239-44.

24 Semrau M, Evans-Lacko S, Alem A, Ayuso-Mateos JL, Chisholm D, Gureje O, et al. Strengthening mental health systems in low- and middle-income countries: the Emerald Programme. BMC Med 2015; 13: 79

25 Charfi F, Ouali U, Spagnolo J, Belhadj A, Nacef F, Saidi O, et al. The mental health system in Tunisia: an overview of services, facilities, and human resources. $J$ Ment Health 2020 [under review].
26 Ministry of Health. The National Strategy for the Promotion of Mental Health. World Health Organization, 2013 (https://www.mindbank.info/item/6277).

27 Spagnolo J, Champagne F, Leduc N, Melki W, Piat M, Laporta M, et al. "We find what we look for, and we look for what we know": factors interacting with a mental health training program to influence its expected outcomes in Tunisia. BMC Public Health 2018; 18(1): 1398.

28 Heim E, Henderson C, Kohrt BA, Koschorke M, Milenova M, Thornicroft G. Reducing mental health-related stigma among medical and nursing students in low- and middle-income countries: a systematic review. Epidemio Psychiatr Sci 2019; 19: e28.

29 Knaak S, Mantler E, Szeto A. Mental illness-related stigma in healthcare: barriers to access and care and evidence-based solutions. Healthc Manage Forum 2017; 30(2): 111-6.

30 Merhej R. Stigma on mental illness in the Arab world: beyond the socio-cultural barriers. Int J Hum Rights Health 2019; 12(4): 285-98.

31 Ungar T, Knaak S, Szeto CHA. Theoretical and practical considerations for combating mental illness stigma in health care. Comm Ment Health J 2016; 52(2): 262-71.

32 Ministère de la santé publique. Décret n̊ 2011-4132 du 17 novembre 2011, fixant le cadre général du régime des études médicales habilitant à l'exercice de la médecine de famille et à la spécialisation en médecine. JORT 2011; 90: 2701-

33 Vigo D, Patten S, Pajer K, Krausz M, Taylor S, Rush B, et al. Mental health of communities during the COVID-19 pandemic. Can J Psychiat [Epub ahead of print] 11 May 2020. Available from: http://doi.org/10.1177/ 0706743720926676

34 Hoeft TJ, Fortney JC, Patel V, Unützer J. Task-sharing approaches to improve mental health care in rural and other low-resource settings: a systematic review. J Rural Health 2018; 34(1): 48-62.

35 Kakuma R, Minas H, Dal Poz MR. Strategies for strengthening human resources for mental health. In Global Mental Health: Principles and Practice (eds V Patel, H Minas, A Cohen, M Prince). Oxford University Press, 2014

36 Curry L, Purkis IA. Validity of self-reports of behavior changes by participants after a CME course. J Med Educ 1986; 61(7): 578-84. 